\title{
MicroRNA-629-5p promotes osteosarcoma proliferation and migration by targeting caveolin 1
}

\author{
Chunsheng $\mathrm{Gao}^{1,2 \odot}$, Jun $\mathrm{Gao}^{2 \odot}$, Ge Zeng ${ }^{2 \oplus}$, Huichao Yan ${ }^{2 \oplus}$, Junhua Zheng ${ }^{2 \oplus}$, and \\ Weichun $\mathrm{Guo}^{30}$ \\ ${ }^{1}$ Department of Orthopedics, Renmin Hospital of Wuhan University, Wuhan, Hubei, China \\ ${ }^{2}$ Department of Orthopedics, The Third People's Hospital of Hubei Province, Wuhan, Hubei, China \\ ${ }^{3}$ Department I of Orthopedics, Renmin Hospital of Wuhan University, Wuhan, Hubei, China
}

\begin{abstract}
Osteosarcoma is a highly malignant tumor that occurs in the bone. Previous studies have shown that multiple microRNAs (miRNAs) regulate the development of osteosarcoma. This study aimed to explore the role of miR-629-5p and its target gene, caveolin 1 (CAV1), in osteosarcoma development. To analyze the expression of miR-629-5p and CAV1 mRNA in osteosarcoma tissues and cell lines, qRT-PCR analysis was performed. Dual-luciferase reporter experiments were subsequently performed to validate the relationship between CAV1 and miR-629-5p. CCK8 assay was used to measure osteosarcoma cell proliferation, and wound-healing assay was performed to study their migratory phenotype. Our findings revealed that miR-629-5p was overexpressed in osteosarcoma tissues and cells, and thereby enhanced cell proliferation and migration. Further, we validated that miR-629-5p targets CAV1 mRNA directly. CAV1 expression, which was negatively correlated with miR-629-5p expression, was found to be downregulated in osteosarcoma tissue samples. Moreover, our data showed that an increase in CAV1 level led to a decline in osteosarcoma cell proliferation and migration, which could be rescued by miR-629-5p upregulation. Overall, our study confirmed that miR-629-5p promoted osteosarcoma proliferation and migration by directly inhibiting CAV1.
\end{abstract}

Key words: Osteosarcoma; miR-629-5p; CAV1; Proliferation; Migration

\section{Introduction}

Osteosarcoma is a malignant bone tumor that typically develops in the knees (1). Osteosarcoma is the most common type of bone cancer in children and adolescents under the age of 20 , and is a life-threatening cancer $(2,3)$. In recent years, advances in diagnostics and treatments have considerably improved the survival rate and quality of life of patients with osteosarcoma (4). Much progress has also been made in decreasing osteosarcoma symptoms owing to the application of chemotherapy, radiotherapy, and new anti-tumor therapies (5-7). However, the cure rate of high-risk osteosarcoma remains low due to drug resistance and metastasis (1). These continue to be major challenges in the successful treatment of osteosarcoma. Therefore, there is an urgent need for further research to identify new treatment modalities for osteosarcoma.

Mature microRNAs (miRNAs) are formed by splicing of precursors encoded by endogenous genes, and many studies have shown that although miRNAs are not directly involved in translation, they have important biological roles such as in the formation of silencing complexes $(8,9)$. As gene regulators, miRNAs induce mRNA degradation or inhibit translation $(10,11)$. Some studies suggest that miRNAs function as oncogenes or tumor suppressor genes in metastasis and development of human cancers (12-14). A growing body of research suggests that multiple miRNAs are involved in osteosarcoma tumorigenesis $(15,16)$. A previous study found that abnormally reduced miR-629-5p expression markedly stimulated the aggressiveness of colorectal cancer (17). In another study, miR-629-5p was identified as a regulator of the development and metastasis of hepatocellular carcinoma (18). Moreover, previous studies have shown that high miR-629 expression predicts poor prognosis and promotes cell proliferation, migration, and invasion in osteosarcoma (19). Nonetheless, the downstream target genes of miR629-5p have not yet been fully investigated.

Caveolin1 (CAV1) constitutes the main structural protein component of caveolae that play a crucial role in various biological processes by interacting with various proteins and non-protein molecules $(20,21)$. Previous studies have shown that inhibition of CAV1 significantly increased the resistance of ovarian cells to paclitaxel (22).

Correspondence: Weichun Guo: <guoweichun86@163.com> 
CAV1 inhibited the proliferation, migration, and invasion of gastric cancer cells (23). In osteosarcoma, CAV1 was found to be significantly decreased in high-grade osteosarcoma compared to normal controls (24). In another study, high metastatic mouse osteosarcoma FBJ-S1 cells were found to have lower expression of CAV1 compared to low metastatic FBJ-LL cells (25). Another study reported the negative effect of CAV1 on osteosarcoma cells by reducing cell proliferation and invasion (26). Taken together, these results indicate that CAV1 acts as a tumor suppressor in osteosarcoma. Bioinformatics analysis revealed that low expression of CAV1 was a key regulator of osteosarcoma and may be the target gene of miR-629-5p.

As the relationship between CAV1 and miR-629-5p in osteosarcoma has not been examined previously, this study aimed to identify the roles of $m i R-629-5 p$ and its potential target gene, CAV1, in osteosarcoma cell phenotypes. It also aimed to determine the underlying molecular mechanisms of osteosarcoma development. The findings of our study may provide further insight into potential molecular targets for osteosarcoma treatments.

\section{Material and Methods}

\section{Tissue acquisition and cell culture}

All osteosarcoma tissue samples used in this study were collected from the Renmin Hospital of the Wuhan University, and the study protocol (approval No. WDRY2015-K419) was approved by the Ethics Committee of the Renmin Hospital of the Wuhan University.

Table 1. Clinical parameters of patients with osteosarcoma in this study.

\begin{tabular}{lr}
\hline Clinical parameters & $\mathrm{n}(\%)$ \\
\hline Gender & \\
Male & $8(42.1 \%)$ \\
Female & $11(57.9 \%)$ \\
Age & \\
$\geqslant 25$ & $10(53.6 \%)$ \\
$<25$ & $9(47.4 \%)$ \\
Location & \\
Distal of femur & $9(47.4 \%)$ \\
Proximal of tibia & $8(42.1 \%)$ \\
Other & $2(10.5 \%)$ \\
Enneking stage & \\
I & $10(52.6 \%)$ \\
II & $6(31.6 \%)$ \\
III & $3(15.8 \%)$ \\
TNM & \\
I+ II & $14(73.7 \%)$ \\
III + IV & $5(26.3 \%)$ \\
Distant metastasis & \\
Negative & $16(84.2 \%)$ \\
Positive & $3(15.8 \%)$ \\
\hline
\end{tabular}

The clinical parameters of osteosarcoma patients are shown in Table 1. Osteosarcoma cell lines (HOS, Saos2, U2OS, and SJSA-1) and immortalized osteoblast line (hFOB1.19) were purchased from the American Type Culture Collection (USA). Cells were cultured under $5 \% \mathrm{CO}_{2}$ at $37^{\circ} \mathrm{C}$ in Dulbecco's modified eagle medium (cat. No. 11320033; Gibco; Thermo Fisher Scientific Inc., USA) with $10 \%$ fetal bovine serum (cat. No. 16140071; Gibco; Thermo Fisher Scientific Inc.) and $100 \mathrm{U} / \mathrm{mL}$ streptomycin (cat. No. 85886; Sigma, China).

\section{Quantitative reverse transcription-polymerase chain reaction (qRT-PCR)}

Total RNA was isolated using TRIzol reagent (cat. No. 15596026; Thermo Fisher Scientific Inc.). Then, the isolated RNAs were reverse transcribed into cDNA. miR$629-5 p$ was reverse transcribed using the mirVana qRTPCR miRNA Detection kit (cat. No. AM1558; Thermo Fisher Scientific Inc.) according to the manufacturer's instructions. CAV1 was reverse transcribed using SuperScript $^{\mathrm{TM}}$ III First-Strand Synthesis SuperMix for qRT-PCR (cat. No. 11752050; Thermo Fisher Scientific Inc.). qRTPCR was then performed using the StepOnePlus ${ }^{\mathrm{TM}}$ RealTime PCR System (cat. No. 4376600; Thermo Fisher Scientific Inc.). The data were analyzed using the $2^{-\Delta \Delta C t}$ method, with U6 (NR_004394.1) and $\beta$-actin (241bp, NM 001172897.2) serving as internal controls for miR-629-5p (miRBase ID: hsa-miR-629-5p, MIMAT0004810) and CAV1 (135 bp, NM_205518.1), respectively. Primer sequences are shown in Table 2.

\section{Cell transfection}

miR-629-5p mimic, miR-629-5p inhibitor, and miR-629$5 p$ negative control were purchased from Guangzhou RiboBio Co., Ltd. (China) (Supplementary Table S1). CAV1overexpression (CAV1 OE) plasmid was constructed by cloning full length CAV1 gene into pcDNA3.1 vector (GeneCopoeia Inc., cat. No. T2806; China). Next, HOS

Table 2. Primer sequences used in this study.

\begin{tabular}{|c|c|}
\hline Primer & Sequences \\
\hline \multicolumn{2}{|l|}{ miR-629-5p } \\
\hline Forward & 5'-TGGGTTTACGTTGGGAGA-3' \\
\hline Reverse & 5'-GTGCAGGGTCCGAGGTATTC-3' \\
\hline \multicolumn{2}{|l|}{ CAV1 } \\
\hline Forward & 5'-CTGTCGGAGCGGGACATCT-3' \\
\hline Reverse & 5'-GCCTTCCAAATGCCGTCAAA-3' \\
\hline \multicolumn{2}{|l|}{$\beta$-actin } \\
\hline Forward & 5'-CACCATTGGCAATGAGCGGTTC-3' \\
\hline Reverse & 5'-AGGTCTTTTGCGGATGTCCACGT-3' \\
\hline \multicolumn{2}{|l|}{ U6 } \\
\hline Forward & 5'-TGCGGGTGCTCGCTTCGGCAGC-3' \\
\hline Reverse & 5'-CCAGTGCAGGGTCCGAGGT-3' \\
\hline
\end{tabular}


and Saos 2 cells were transiently transfected with $75 \mathrm{nM}$ miR-629-5p mimic, negative control, miR-629-5p inhibitor, and $3 \mu \mathrm{g}$ CAV1 OE plasmid using Lipofectamine 2000 (cat. No. 11668019; Thermo Fisher Scientific Inc.) at room temperature. After the cells were incubated for 2 days at $37^{\circ} \mathrm{C}$, they were analyzed by qRT-PCR.

\section{CCK-8 assay}

Cells $(100 \mu \mathrm{L})$ suspended in logarithmic growth phase were plated in 96 -well plates (2000 cells/well) and cultured at $37^{\circ} \mathrm{C}$ for the appropriate duration of 12 , 24, 48, and $72 \mathrm{~h}$. Then, $10 \mu \mathrm{L}$ CCK-8 solution was added to each well according to the CCK-8 user manual (cat. No. E606335; Sangon, China). After the cells were incubated with CCK-8 for $2 \mathrm{~h}$, the absorbance was measured at $450 \mathrm{~nm}$.

\section{Wound-healing assay}

Cells $\left(2 \times 10^{5}\right.$ cells/well) were plated in 6 -well plates and cultured until they reached $80-90 \%$ confluence. The cell monolayer was scratched in a straight line with a sterile $200 \mu \mathrm{L}$ pipette tip to simulate a wound, and the scraped off cells were removed. The medium was replaced with serum-free media containing $10 \mu \mathrm{g} / \mathrm{mL}$ of mitomycin- $C$ to stop proliferation. The cells in the blank control (CON) group were not treated with mitomycin-C. Cells were photographed at 0 and $24 \mathrm{~h}$. The migrated distance (i.e., scratch width at $0 \mathrm{~h}$ - scratch width at $24 \mathrm{~h}$ ) from the edge of the scratch was measured using Adobe Illustrator, a software application (USA).

\section{Bioinformatics analysis}

GSE11414 and GSE12865 data series were downloaded from the gene expression omnibus (GEO) database (https://www.ncbi.nlm.nih.gov/gds/?term=). GSE11414 (27) included four osteosarcoma cell samples (two MG63 cell line samples and two U2OS cell line samples) and two normal human osteoblasts samples (two HOB cell line samples). GSE12865 (28) included twelve osteosarcoma tissue samples and two primary normal human osteoblast cell samples, which were obtained from healthy male donors. The two data series were analyzed using the GEO2R algorithm built into the GEO database (https:// www.ncbi.nlm.nih.gov/geo/). The differentially expressed genes (DEGs) in GSE11414 were identified using the criteria of adjusted $\mathrm{P}<0.05$ and $|\log \mathrm{FC}| \geqslant 1.5$, whereas those in GSE12865 were identified using the criteria of adjusted $\mathrm{P}<0.05$ and $|\log \mathrm{FC}| \geqslant 2$. TargetScan Human 7.2, an online tool (http://www.targetscan.org/vert_72/), was used to predict potential target genes of miR-629$5 p$. Then, the overlapping genes from GSE12865 DEGs, GSE11414 DEGs, and the target genes of miR-629-5p by Venny 2.1.0 (https://bioinfogp.cnb.csic.es/tools/venny/) were selected. Finally, the overlapping genes were uploaded to the STRING algorithm (https://string-db.org/) to identify the key gene.

\section{Luciferase reporter assay}

First, full length CAV1 $3^{\prime}$ UTR (wild-type) or its mutant (CAV1-MUT, 205-211 sequence) were cloned into psiCHECK-2 vector (GenePharma, China). HOS and Saos2 cells were then co-transfected with miR-629-5p mimic and wild-type CAV1 or CAV1-MUT luciferase reporter plasmids. After $48 \mathrm{~h}$ of incubation, the culture medium was removed and cells were collected. The collected cells were lysed to generate cell lysates. Luciferase activity was measured using the Pierce ${ }^{\mathrm{TM}}$ Renilla-Firefly Luciferase Dual Assay kit (cat. No. 16185; Thermo Fisher Scientific Inc.) according to the manufacturer's guidelines.

\section{Statistical analysis}

All means and standard deviations were calculated based on three independent experiments. One-way analysis of variance (ANOVA) was used for statistical analysis between multiple groups. Mann-Whitney test was used for statistical analysis of RNA expression in tissue samples. $\mathrm{P}<0.05$ was considered statistically significant.

\section{Results}

\section{Upregulation of $m i R-629-5 p$ in osteosarcoma}

Examination of $\mathrm{U} 6 \mathrm{C}_{\mathrm{T}}$ values in osteosarcoma tissues and cell lines revealed no significant difference in the $C_{T}$ values between that of osteosarcoma and adjacent normal tissues. Furthermore, we observed that the U6 $\mathrm{C}_{\mathrm{T}}$ values of HOS, U2OS, SJSA-1, and Saos2 were similar to those of hFOB1.19 (Supplementary Figure S1A and $B$ ). These results showed that U6 was stably expressed in osteosarcoma tissues and cell lines. Thus, U6 was used as the internal reference for $m i R-629-5 p$ in subsequent experiments. miR-629-5p expression was analyzed in osteosarcoma $(n=19)$ and adjacent normal tissues $(n=19)$ using qRT-PCR. The relative expression level of $m i R-629-5 p$ was significantly higher in osteosarcoma tissues than that in the adjacent healthy tissues $(P=0.0002)$ (Figure 1A). We also observed that osteosarcoma cell lines (HOS, U2OS, SJSA-1, and Saos2) displayed higher expression levels of miR-629-5p compared to the immortalized osteoblast cell line hFOB1.19 (Figure 1B). In addition, Saos2 and HOS cell lines showed higher miR-629-5p expression levels compared to the other osteosarcoma cell lines $(P<0.001)$. Therefore, Saos2 and HOS cells were selected for subsequent experiments. In summary, miR-629-5p was upregulated not only in osteosarcoma tissues but also in cell lines.

\section{Effect of $m i R-629-5 p$ in osteosarcoma}

The qRT-PCR analysis revealed that transfection with the miR-629-5p mimic markedly increased the expression of miR-629-5p, while transfection with the miR-629-5p inhibitor reduced miR-629-5p expression. These results indicated successful transfection of both cell lines 

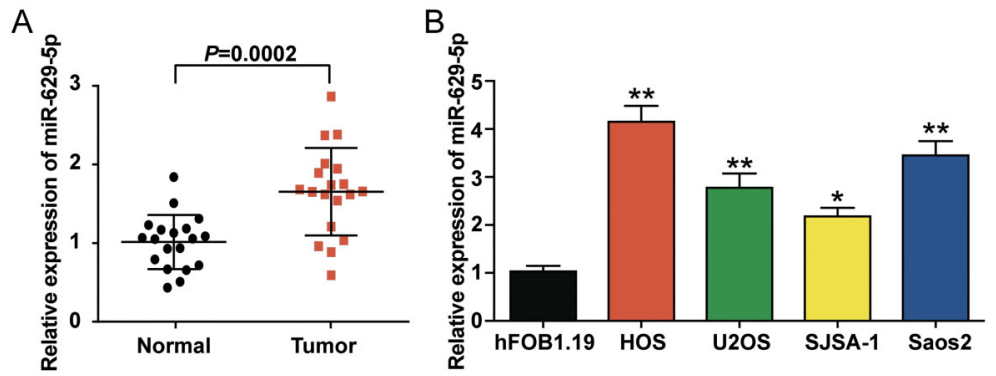

Figure 1. miR-629-5p was upregulated in osteosarcoma tissues and cells. A, The relative expression level of miR-629-5p was analyzed using qRT-PCR in osteosarcoma $(n=19)$ and adjacent healthy tissues $(n=19)$. miR-629-5p was upregulated in osteosarcoma tissues. U6 was used as the internal control. B. The expression of miR-629-5p in osteosarcoma cell lines (HOS, U2OS, SJSA-1, and Saos2) and an immortalized normal osteocyte cell line (hFOB1.19) was determined by qRT-PCR. U6 was used as the internal control. Data are reported as mean $\pm \mathrm{SD}$. ${ }^{*} \mathrm{P}<0.05,{ }^{* *} \mathrm{P}<0.001$ compared with $\mathrm{hFOB} 1.19$ cells (ANOVA).

$(P<0.001)$ (Figure $2 A)$. The CCK-8 assay was used to evaluate the rate of proliferation of the transfected cells. CCK-8 results showed that upregulation of miR-629-5p significantly enhanced the proliferation of Saos2 and HOS cells, while downregulation of miR-629-5p markedly weakened the proliferation rate $(P<0.001)$ (Figure 2B). In addition, miR-629-5p upregulation increased cell migration $(\mathrm{P}<0.05)$, whereas miR-629-5p downregulation inhibited it $(\mathrm{P}<0.001)$ (Figure $2 \mathrm{C})$. These results confirmed that miR-629-5p significantly increased the malignant phenotypes of osteosarcoma cells.

\section{CAV1 was a target of miR-629-5p}

To identify the potential downstream interacting targets of miR-629-5p that participate in osteosarcoma pathogenesis, we downloaded two GEO expression datasets (GSE12865 and GSE11414) and analyzed the common differentially expressed genes (DEGs) (Supplementary Figure S2A and B). We found 81 common genes that were significant DEGs in osteosarcoma, including those with potential binding targets of $m i R$ $629-5 p$ (Figure 3A). We hypothesized that these 81 genes may have significant roles in osteosarcoma progression. Therefore, we used the STRING algorithm to analyze the interaction between the 81 genes. The results showed that CAV1 had the greatest number of, and the most evident interactions with, other genes in the network (Figure 3B). Therefore, we selected CAV1 for further analysis and retrieved the binding sequence between miR-629-5p and CAV1 from TargetScan Human 7.2 (Figure $3 \mathrm{C}$ ). To further ascertain the relationship between miR-629-5p and CAV1, we mutated the $3^{\prime}$ UTR sequence of CAV1 that binds to miR-629-5p and constructed luciferase reporter plasmid (CAV1-MUT). miR-629-5p mimic and the luciferase reporter plasmid containing wild-type or mutated CAV1 mRNA 3'UTR were co-transfected into Saos 2 and HOS cells. Cotransfection of miR-629-5p mimic with wild-type CAV1
mRNA 3'UTR that contained the binding site of miR-629$5 p$ showed reduced luciferase activity, while co-transfection of miR-629-5p mimic and CAV1 mutant did not show decreased luciferase activity in either cell line $(P<0.001)$ (Figure 3D). Next, we analyzed the expression of CAV1 mRNA in the tissue samples collected. Although only a limited number of osteosarcoma samples were analyzed, we found that CAV1 mRNA was significantly downregulated in the osteosarcoma tissues compared to adjacent healthy tissues $(P=0.0009)$ (Figure 3E). In addition, a negative correlation was identified between miR-629-5p and CAV1 expression in osteosarcoma tissues $(P<0.001)$ (Figure $3 F)$. We also analyzed the expression of CAV1 mRNA in osteosarcoma cell lines. The results showed that CAV1 was significantly downregulated in osteosarcoma cell lines $(P<0.05)$ (Figure $3 G)$. These results indicated that CAV1 may be a potential effector in osteosarcoma and a downstream target of $m i R-629-5 p$.

\section{Influence of $m i R-629-5 p$ on osteosarcoma cell lines by targeting CAV1}

Analyses revealed that CAV1 OE plasmid transfection increased CAV1 levels but did not affect miR-629-5p expression in either cell line. Transfection of $m i R-629-5 p$ mimic increased miR-629-5p but decreased CAV1 $(\mathrm{P}<0.001)$ (Figure 4A). Thus, the $q R T-P C R$ results indicated successful cell transfection. As shown in Figure 4B, CAV1 upregulation reduced the proliferation of Saos2 and HOS cell lines that was restored by upregulation of miR-629-5p $(\mathrm{P}<0.001)$. Wound-healing assay demonstrated that CAV1-upregulated cells showed impaired migration, but upregulation of miR-629-5p significantly restored the reduction in cell migration caused by CAV1 overexpression $(P<0.001)$ (Figure $4 C)$. These results suggested that miR-629-5p promoted the malignant phenotypes in osteosarcoma cell lines by directly inhibiting CAV1. 
A
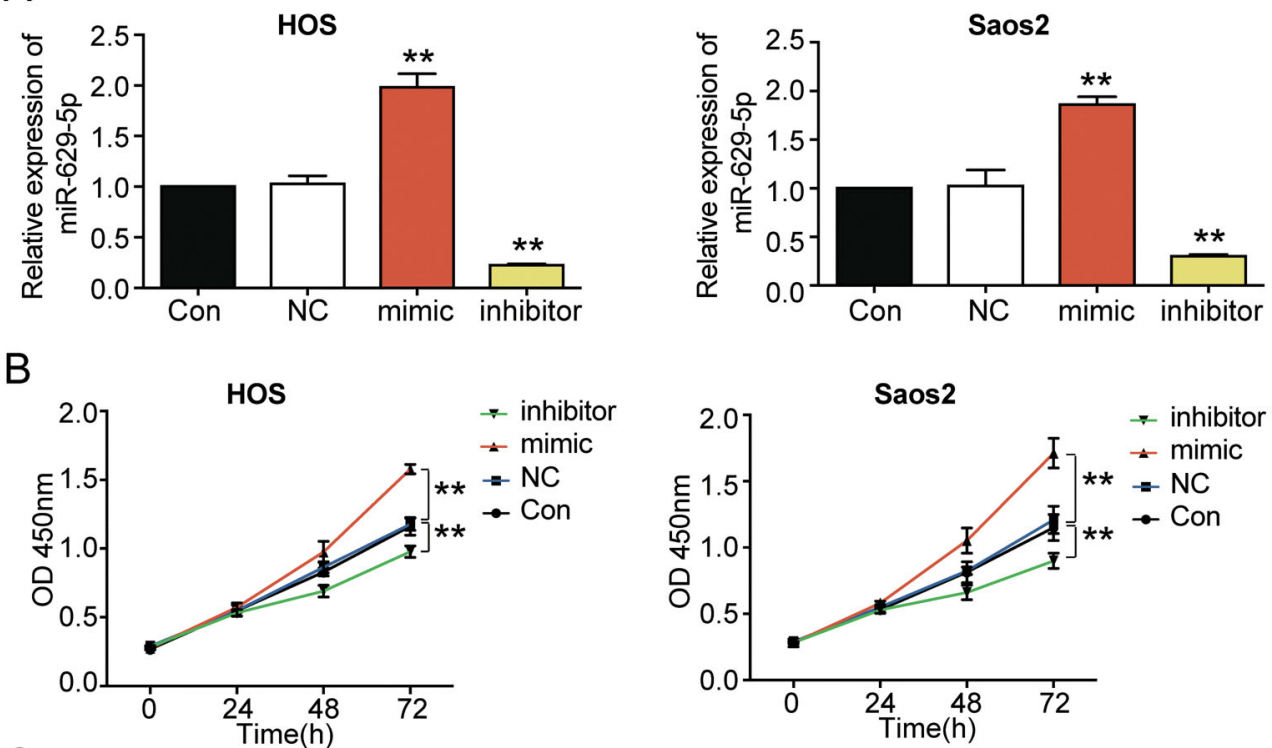

C
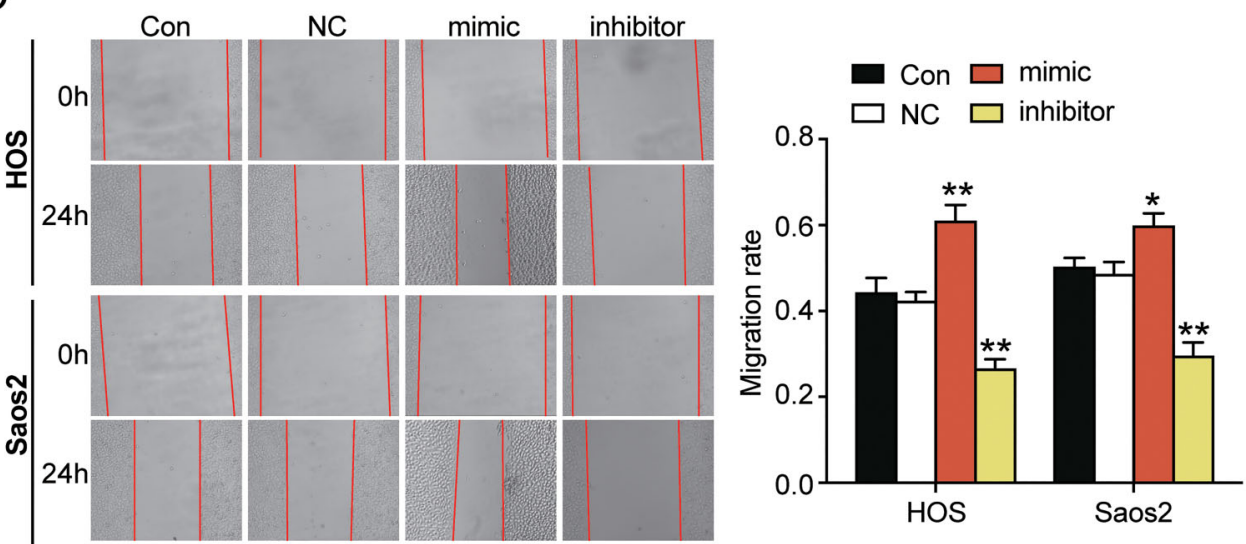

Figure 2. miR-629-5p upregulation promoted proliferation and migration of HOS and Saos2 cell lines. A, miR-629-5p mimic transfection significantly increased miR-629-5p expression, while miR-629-5p inhibitor significantly reduced it, thus indicating successful cell transfection. B, After the miR-629-5p mimic or inhibitor was transfected into HOS and Saos2 cells, the proliferation was determined using CCK-8 assay. C, The migration phenotypes of the HOS and Saos2 cells transfected with miR-629-5p mimic or inhibitor were detected with wound-healing assay. CON: blank control; NC: negative control; Mimic: miR-629-5p mimic; Inhibitor: miR-629-5p inhibitor. A-C, The data of the three independent experiments are reported as means $\pm \mathrm{SD}$. ${ }^{*} \mathrm{P}<0.05,{ }^{* *} \mathrm{P}<0.001$ compared with control group (ANOVA).

\section{Discussion}

In this study, we demonstrated that miR-629-5p and CAV1 played crucial roles in osteosarcoma development. Specifically, the upregulation of miR-629-5p in osteosarcoma tissues and cells promoted osteosarcoma cell proliferation and migration. In addition, CAV1 was downregulated in osteosarcoma tissues and cell lines, and its overexpression reduced osteosarcoma cell proliferation and migration. Our results showed that an increase in miR-629-5p promoted the malignant phenotype of osteosarcoma cell lines by directly suppressing CAV1.
miR-629-5p upregulation has been found in various cancers (cervical cancer, lymphoblastic leukemia, and colorectal cancer), and it is used as a biomarker for monitoring disease progression (29-31). An abnormal increase in miR-629-5p levels stimulates cell proliferation, migration, and invasion and accelerates the aggressiveness of hepatocellular carcinoma cells, thereby resulting in tumor growth and metastasis (18). Interestingly, a study showed that miR-629-5p was associated with tipifarnib resistance in breast cancer cells (32). In another study, high levels of miR-629-5p in HPV-positive cervical cancer cells were associated with cancer-promoting activities that 
A

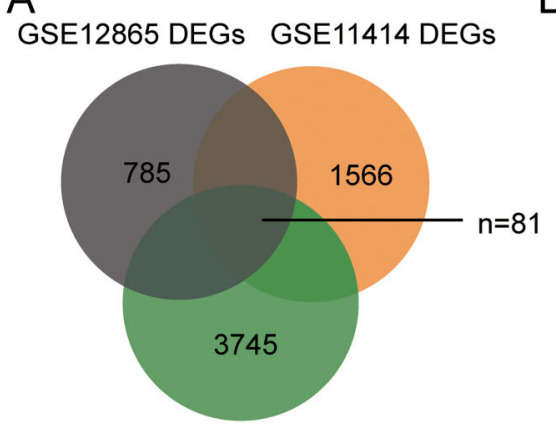

TargetScan Human 7.2

C

Position 205-211 of CAV1 3'UTR

hsa-miR-629-5p

D

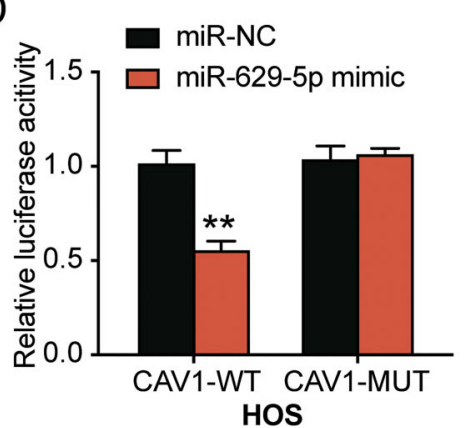

F

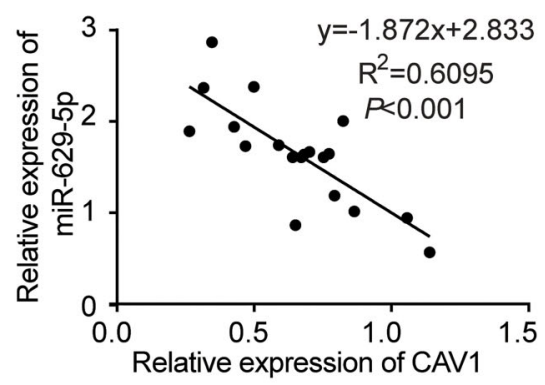

B

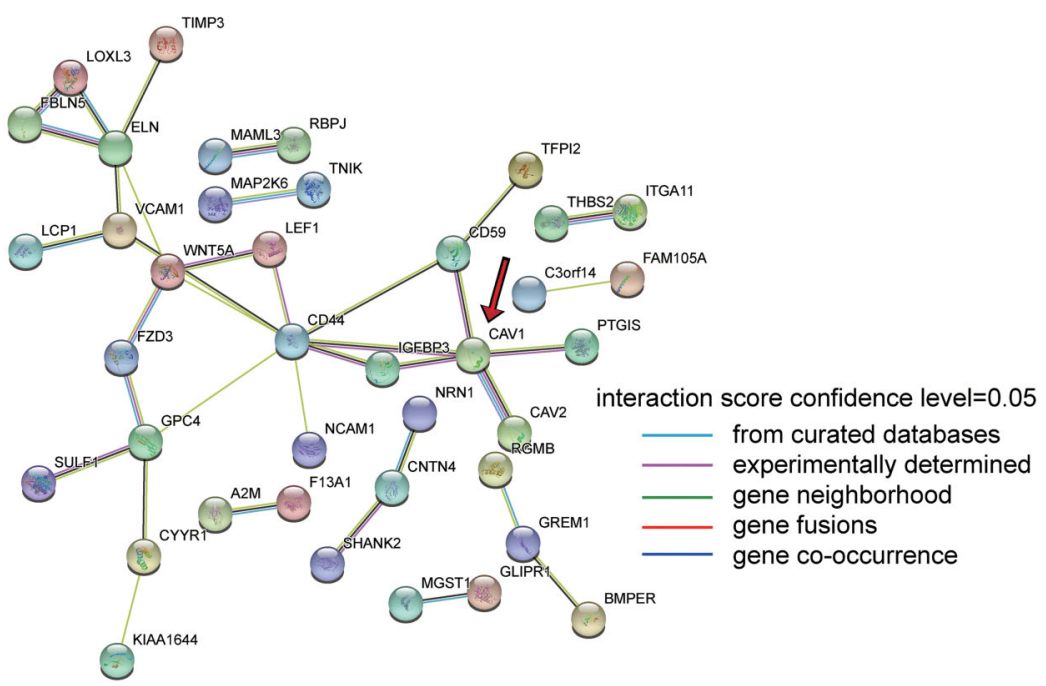

5'...cagucugaauuuuuaAAACCC-Au...

3' caagaggguugcaUUUGGGU

$\mathrm{E}$
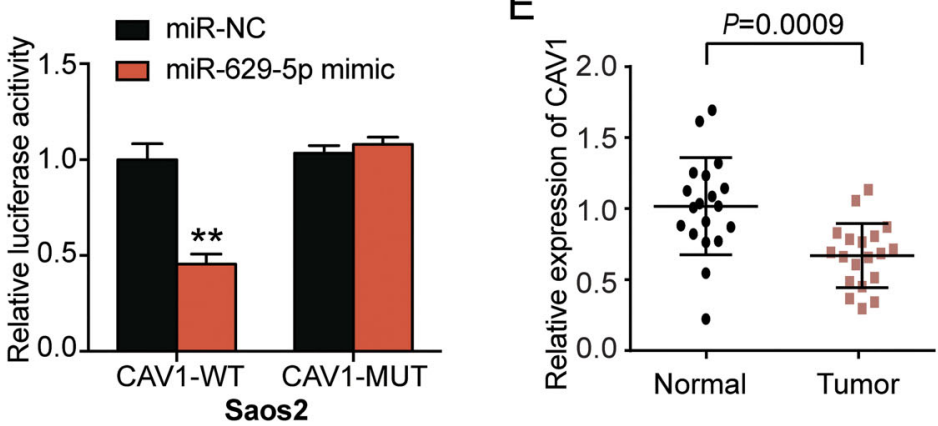

$\mathrm{G}$

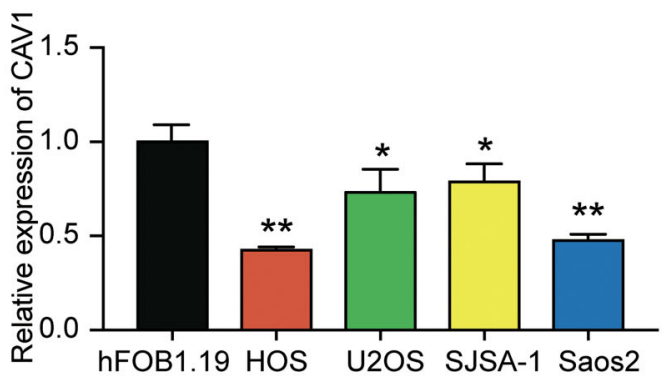

Figure 3. miR-629-5p directly targeted CAV1. A, Intersection between differentially expressed genes (DEGs) from GSE11414 and GSE12865 and the predicted targets of miR-629-5p by TargetScan Human 7.2. B, STRING interrogation output of the 81 common genes from the last step. C, TargetScan Human 7.2 was used to predict the potential binding site between miR-629-5p and CAV1. D, Potential binding between miR-629-5p and 3'UTR of CAV1 was validated by dual-luciferase reporter assay in HOS and Saos2 cell lines. miR-629-5p mimic attenuated the fluorescent activity of CAV1-WT but did not affect the fluorescent activity of CAV1-MUT. ${ }^{* *} \mathrm{P}<0.001$ compared with the co-transfection of the CAV1-WT group and the NC group. $\mathrm{E}$, The expression level of CAV1 in osteosarcoma tissues and normal tissues was measured by qRT-PCR. F, The relationship between miR-629-5p and CAV1 was identified using Pearson correlation analysis. G, The expression of CAV1 in osteosarcoma cell lines and hFOB1.19 cell line. $\beta$-actin was used as the internal control. CAV1-WT: wild-type CAV1; CAV1-MUT, CAV1 mutant; NC: negative control. ${ }^{*} \mathrm{P}<0.05$, ${ }^{\star \star} \mathrm{P}<0.001$ compared with hFOB1.19 cells (ANOVA). $\mathbf{D}$ and $\mathbf{G}$, The data of the three independent experiments are reported as means \pm SD. 
A
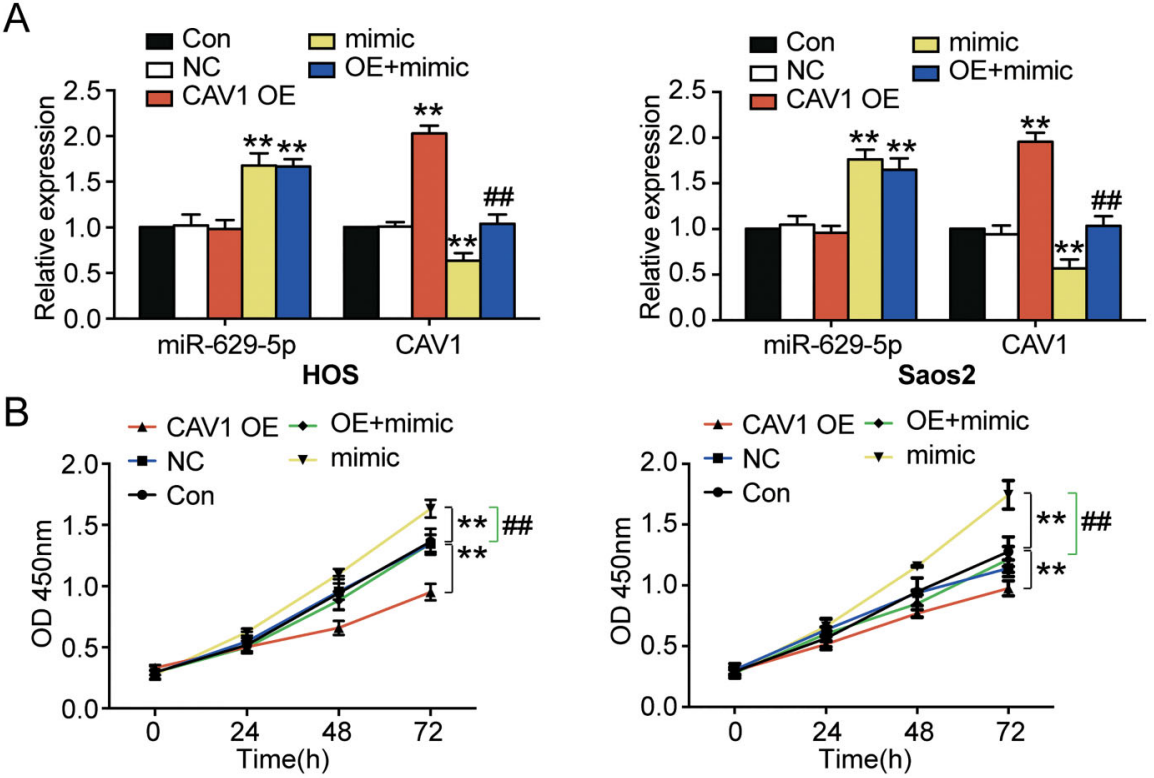

HOS

Saos2
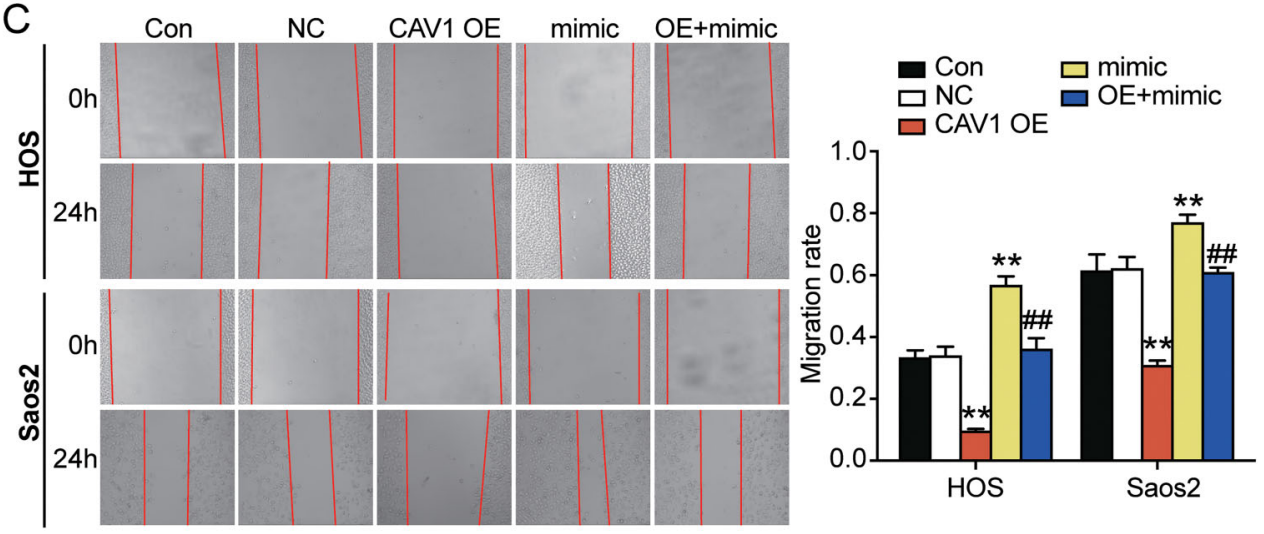

Figure 4. miR-629-5p rescued the inhibitory effect of CAV1 over-expression on osteosarcoma cell phenotypes. A, The transfection efficiency of miR-629-5p mimic and CAV1 overexpression plasmids in transfected cells was detected by qRT-PCR. B, After transfection with miR-629-5p mimic or CAV1 OE, the proliferation of transfected Saos2 or HOS cells was determined with CCK-8 assay. C, The migration rate of the Saos2 or HOS cells transfected with miR-629-5p mimic or CAV1 OE was identified using wound-healing assay. CON: blank control; NC: negative control; mimic: miR-629-5p mimic; CAV1 OE: CAV1 over-expression. The data of the three independent experiments are reported as means $\pm S D$. ${ }^{* *} \mathrm{P}<0.001$ compared with the control group. ${ }^{\#} \mathrm{P}<0.001$ compared with the CAV1 OE group (ANOVA).

enhanced E6/E7-dependent cell proliferation and simultaneously reduced apoptosis (33). Furthermore, aberrantly elevated miR-629-5p promoted proliferation while inhibiting apoptosis, thus exacerbating and aggravating ovarian cancer (34). In this study, we found that miR-629-5p that increased the aggressiveness of osteosarcoma cells was upregulated during the growth of osteosarcoma.

In addition, studies have reported that miR-629-5p may potentially promote various oncogenic processes through miRNA-mRNA regulatory networks. In one study, miR-629-5p was found to enhance carcinogenesis of human hepatocellular carcinoma by targeting SFRP2 (18). Another study demonstrated that miR-629-5p contributes to ovarian cancer by directly suppressing downstream target genes, including FGF1, AKT3, or MAGI2 (35). Moreover, miR-629-5p was demonstrated to drive the generation of more aggressive colorectal cancer cells by directly downregulating $C X X C 4$, which affected 5-FU sensitivity (31). Further, the downregulation of miR-629-5p inhibits ovarian cancer by targeted inhibition of TSPYL5 (34). Consistent with these findings, our study found that miR-629-5p facilitated the pathological process of 
osteosarcoma by directly inhibiting the expression of the target gene, CAV1. Collectively, these data suggest that miR-629-5p may participate in tumorigenesis by downregulating the expression of certain molecules.

A previous study found that abnormal expression of CAV1 is associated with the degree of malignancy of osteosarcoma cells, with CAV1 downregulation in highly malignant cells (36). In this study, we found that CAV1 was downregulated in osteosarcoma tissues. To date, inactivation of CAV1 has been shown to be associated with the occurrence of multiple types of malignancies (37). In addition, CAV1 was shown to reduce paclitaxel resistance in osteosarcoma cells by attenuating PI3K-Akt-JNKdependent autophagy (38). In this study, we found that CAV1 overexpression repressed the malignant phenotype in osteosarcoma cells. Our results were similar to a study that showed that CAV1 inhibited osteosarcoma cell (Saos-2 cell line) proliferation and invasion by upregulating the calcium sensing-receptor CaSR (39). Another study found that CAV1 that suppresses the progression of

\section{References}

1. Isakoff MS, Bielack SS, Meltzer P, Gorlick R. Osteosarcoma: current treatment and a collaborative pathway to success. J Clin Oncol 2015; 33: 3029-3035, doi: 10.1200/JCO.2014. 59.4895.

2. Whelan J, McTiernan A, Cooper N, Wong YK, Francis M, Vernon S, et al. Incidence and survival of malignant bone sarcomas in England 1979-2007. Int J Cancer 2012; 131: E508-E517, doi: 10.1002/ijc.26426.

3. Bielack SS, Carrle D, Hardes J, Schuck A, Paulussen M. Bone tumors in adolescents and young adults. Curr Treat Options Oncol 2008; 9: 67-80, doi: 10.1007/s11864-0080057-1.

4. Clark JC, Dass CR, Choong PF. A review of clinical and molecular prognostic factors in osteosarcoma. J Cancer Res Clin Oncol 2008; 134: 281-297, doi: 10.1007/s00432-0070330-x.

5. Parsai S, Juloori A, Angelov L, Scott JG, Krishnaney AA, Udo-Inyang I, et al. Spine radiosurgery in adolescents and young adults: early outcomes and toxicity in patients with metastatic Ewing sarcoma and osteosarcoma. J Neurosurg Spine 2019: 1-8.

6. Fathizadeh $\mathrm{H}$, Mirzaei $\mathrm{H}$, Asemi Z. Melatonin: an anti-tumor agent for osteosarcoma. Cancer Cell Int 2019; 19: 319, doi: 10.1186/s12935-019-1044-2.

7. Chen EL, Yoo CH, Gutkin PM, Merriott DJ, Avedian RS, Steffner RJ, et al. Outcomes for pediatric patients with osteosarcoma treated with palliative radiotherapy. Pediatr Blood Cancer 2020; 67: e27967, doi: 10.1002/pbc.27967.

8. Caldas C, Brenton JD. Sizing up miRNAs as cancer genes. Nat Med 2005; 11: 712-714, doi: 10.1038/nm0705-712.

9. Ling $H$, Fabbri $M$, Calin GA. MicroRNAs and other noncoding RNAs as targets for anticancer drug development. Nat Rev Drug Discov 2013; 12: 847-865, doi: 10.1038/ nrd4140. ovarian cancer is directly inhibited by miR-96-5p, indicating that CAV1 may be regulated by miRNAs in carcinogenesis (40).

This study had some limitations. For instance, our study did not explore the downstream regulatory network involving miR-629-5p and CAV1. Animal experiments have not yet been conducted to verify the malignant behavior of tumors. Moreover, clinical analysis is needed to determine whether miR-629-5p has the potential to aggravate tumor metastasis. Nonetheless, our results confirmed that miR-629-5p stimulates the malignant phenotype of osteosarcoma cell lines by directly downregulating CAV1. In addition, our findings suggested that the miR-629-5p-CAV1 interactome represents a potential new target for osteosarcoma treatment.

\section{Supplementary Material}

Click to view [pdf].

10. Xia W, Zhou J, Luo H, Liu Y, Peng C, Zheng W, et al. MicroRNA-32 promotes cell proliferation, migration and suppresses apoptosis in breast cancer cells by targeting FBXW7. Cancer Cell Int 2017; 17: 14, doi: 10.1186/s12935017-0383-0.

11. Iwaki J, Kikuchi K, Mizuguchi $\mathrm{Y}$, Kawahigashi $\mathrm{Y}$, Yoshida $\mathrm{H}$, Uchida E, et al. MiR-376c down-regulation accelerates EGFdependent migration by targeting GRB2 in the HuCCT1 human intrahepatic cholangiocarcinoma cell line. PLoS One 2013; 8: e69496, doi: 10.1371/journal.pone.0069496.

12. Li Y, Chen R, Li Z, Cheng H, Li X, Li T, et al. miR-204 negatively regulates cell growth and metastasis by targeting ROBO4 In human bladder cancer. Onco Targets Ther 2019; 12: 8515-8524, doi: $10.2147 / O T T . S 205023$.

13. Li Y, Xu J, Zhang J, Zhang J, Zhang J, Lu X. MicroRNA-346 inhibits the growth of glioma by directly targeting NFIB. Cancer Cell Int 2019; 19: 294, doi: 10.1186/s12935-0191017-5.

14. Chang M, Yan P, Zhang B, Zhang G, Wang J, Ge H, et al. MicroRNA-769-5p promotes the growth of glioma cells by targeting lysine methyltransferase 2A. Onco Targets Ther 2019; 12: 9177-9187, doi: 10.2147/OTT.S222836.

15. Fellenberg J, Lehner B, Saehr H, Schenker A, Kunz P. Tumor suppressor function of miR-127-3p and miR-376a-3p in osteosarcoma cells. Cancers (Basel) 2019; 11: 2019, doi: 10.3390/cancers 11122019

16. Du K, Zhang X, Lou Z, Guo P, Zhang F, Wang B, et al. MicroRNA485-3p negatively regulates the transcriptional co-repressor CtBP1 to control the oncogenic process in osteosarcoma cells. Int J Biol Sci 2018; 14: 1445-1456, doi: 10.7150/ijbs. 26335.

17. Yan G, Li C, Zhao Y, Yue M, Wang L. Downregulation of microRNA6295p in colorectal cancer and prevention of the malignant phenotype by direct targeting of low density 
lipoprotein receptor related protein 6. Int J Mol Med 2019; 44: 1139-1150, doi: 10.3892/ijmm.2019.4245.

18. Tao X, Yang X, Wu K, Yang L, Huang $Y$, Jin Q, et al. miR629-5p promotes growth and metastasis of hepatocellular carcinoma by activating beta-catenin. Exp Cell Res 2019; 380: 124-130, doi: 10.1016/j.yexcr.2019.03.042.

19. Li X, Li N, Niu Q, Zhu H, Wang Z, Hou Q. Elevated expression of miR-629 predicts a poor prognosis and promotes cell proliferation, migration, and invasion of osteosarcoma. OncoTargets Ther 2020; 13: 1851-1857, doi: $10.2147 / O T T . S 232479$.

20. Cohen AW, Hnasko R, Schubert W, Lisanti MP. Role of caveolae and caveolins in health and disease. Physiol Rev 2004; 84: 1341-1379, doi: 10.1152/physrev.00046.2003.

21. Chidlow JH Jr, Sessa WC. Caveolae, caveolins, and cavins: complex control of cellular signalling and inflammation. Cardiovasc Res 2010; 86: 219-225, doi: 10.1093/cvr/ cvq075.

22. Kanlikilicer $\mathrm{P}$, Bayraktar R, Denizli M, Rashed MH, Ivan C, Aslan $\mathrm{B}$, et al. Exosomal miRNA confers chemo resistance via targeting CAV1/p-gp/M2-type macrophage axis in ovarian cancer. EBioMedicine 2018; 38: 100-112, doi: 10.1016/ j.ebiom.2018.11.004.

23. Luo Z, Rong Z, Zhang J, Zhu Z, Yu Z, Li T, et al. Circular RNA circCCDC9 acts as a miR-6792-3p sponge to suppress the progression of gastric cancer through regulating CAV1 expression. Mol Cancer 2020; 19: 86, doi: 10.1186/s12943020-01203-8.

24. Ning B, Xu DL, Gao JH, Wang LL, Yan SY, Cheng S. Identification of pathway-related modules in high-grade osteosarcoma based on topological centrality of network strategy. Eur Rev Med Pharmacol Sci 2016; 20: 2209-2220.

25. Yang XY, Huang CC, Kan QM, Li Y, Liu D, Zhang XC, et al. Calcium regulates caveolin-1 expression at the transcriptional level. Biochem Biophys Res Commun 2012; 426: 334-341, doi: 10.1016/j.bbrc.2012.08.079.

26. Mohamed FEA, Khali EZI, Toni NDM. Caveolin-1 expression together with VEGF can be a predictor for lung metastasis and poor prognosis in osteosarcoma. Pathol Oncol Res 2020; 26: 1787-1795, doi: 10.1007/s12253-019-00755-5.

27. Sadikovic B, Yoshimoto M, Al-Romaih K, Maire G, Zielenska $\mathrm{M}$, Squire JA. In vitroanalysis of integrated global highresolution DNA methylation profiling with genomic imbalance and gene expression in osteosarcoma. PLOS One2008; 3: e2834, doi: 10.1371/journal.pone.0002834.

28. Sadikovic B, Yoshimoto M, Chilton-MacNeill S, Thorner P, Squire JA, Zielenska M. Identification of interactive networks of gene expression associated with osteosarcoma oncogenesis by integrated molecular profiling. Hum Mol Gen 2009; 18: 1962-1975, doi: 10.1093/hmg/ddp117.

29. Sanchez-Jimenez C, Carrascoso I, Barrero J, Izquierdo JM. Identification of a set of miRNAs differentially expressed in transiently TIA-depleted HeLa cells by genome-wide profiling. BMC Mol Biol 2013; 14: 4, doi: 10.1186/1471-2199-14-4.

30. Almeida RS, Costa ESM, Coutinho LL, Garcia Gomes R, Pedrosa F, Massaro JD, et al. MicroRNA expression profiles discriminate childhood T- from B-acute lymphoblastic leukemia. Hematol Oncol 2019; 37: 103-112, doi: 10.1002/ hon.2567.

31. Lu J, Lu S, Li J, Yu Q, Liu L, Li Q. MiR-629-5p promotes colorectal cancer progression through targetting CXXC finger protein 4. Biosci Rep 2018; 38: BSR20180613, doi: 10.1042/BSR20180613.

32. Uhr K, Prager-van der Smissen WJC, Heine AAJ, Ozturk B, van Jaarsveld MTM, Boersma AWM, et al. MicroRNAs as possible indicators of drug sensitivity in breast cancer cell lines. PLoS One 2019; 14: e0216400, doi: 10.1371/journal. pone. 0216400 .

33. Honegger A, Schilling D, Bastian S, Sponagel J, Kuryshev $V$, Sultmann $H$, et al. Dependence of intracellular and exosomal microRNAs on viral E6/E7 oncogene expression in HPV-positive tumor cells. PLoS Pathog 2015; 11: e1004712, doi: 10.1371/journal.ppat.1004712.

34. Shao L, Shen Z, Qian H, Zhou S, Chen Y. Knockdown of miR-629 inhibits ovarian cancer malignant behaviors by targeting testis-specific Y-like protein 5. DNA Cell Biol 2017; 36: 1108-1116, doi: 10.1089/dna.2017.3904.

35. Wu C, Zhao Y, Liu Y, Yang X, Yan M, Min Y, et al. Identifying miRNA-mRNA regulation network of major depressive disorder in ovarian cancer patients. Oncol Lett 2018; 16: 5375-5382, doi: 10.3892/ol.2018.9243.

36. Zucchini C, Bianchini M, Valvassori L, Perdichizzi S, Benini $S$, Manara MC, et al. Identification of candidate genes involved in the reversal of malignant phenotype of osteosarcoma cells transfected with the liver/bone/kidney alkaline phosphatase gene. Bone 2004; 34: 672-679, doi: 10.1016/ j.bone.2003.12.008.

37. Williams TM, Lisanti MP. Caveolin-1 in oncogenic transformation, cancer, and metastasis. Am J Physiol Cell Physiol 2005; 288: C494-C506, doi: 10.1152/ajpcell.00458.2004.

38. Guan J, Yuan Z, He J, Wu Z, Liu B, Lin X, et al. Overexpression of caveolin-1 reduces Taxol resistance in human osteosarcoma cells by attenuating PI3K-Akt-JNK dependent autophagy. Exp Ther Med 2016; 12: 2815-2822, doi: 10.3892/etm.2016.3713.

39. Jung SY, Kwak JO, Kim HW, Kim DS, Ryu SD, Ko CB, et al. Calcium sensing receptor forms complex with and is upregulated by caveolin-1 in cultured human osteosarcoma (Saos-2) cells. Exp Mol Med 2005; 37: 91-100, doi: 10.1038/emm.2005.13.

40. Liu B, Zhang J, Yang D. miR-96-5p promotes the proliferation and migration of ovarian cancer cells by suppressing Caveolae1. J Ovarian Res 2019; 12: 57, doi: 10.1186/ s13048-019-0533-1. 